\title{
Physical Analysis of the Initial Core and Running-In Phase for Pebble-Bed Reactor HTR-PM
}

\author{
Jingyu Zhang, ${ }^{1} \mathrm{Fu} \mathrm{Li},{ }^{2}$ and Yuliang Sun ${ }^{2}$ \\ ${ }^{1}$ School of Nuclear Science and Engineering, North China Electric Power University, Beijing 102206, China \\ ${ }^{2}$ Institute of Nuclear and New Energy Technology, Tsinghua University, Beijing 100084, China
}

Correspondence should be addressed to Jingyu Zhang; poptnt@163.com

Received 19 December 2016; Revised 9 March 2017; Accepted 23 March 2017; Published 16 April 2017

Academic Editor: Rafael Miró

Copyright (c) 2017 Jingyu Zhang et al. This is an open access article distributed under the Creative Commons Attribution License, which permits unrestricted use, distribution, and reproduction in any medium, provided the original work is properly cited.

\begin{abstract}
The pebble-bed reactor HTR-PM is being built in China and is planned to be critical in one or two years. At present, one emphasis of engineering design is to determine the fuel management scheme of the initial core and running-in phase. There are many possible schemes, and many factors need to be considered in the process of scheme evaluation and analysis. Based on the experience from the constructed or designed pebble-bed reactors, the fuel enrichment and the ratio of fuel spheres to graphite spheres are important. In this paper, some relevant physical considerations of the initial core and running-in phase of HTR-PM are given. Then a typical scheme of the initial core and running-in phase is proposed and simulated with VSOP code, and some key physical parameters, such as the maximum power per fuel sphere, the maximum fuel temperature, the refueling rate, and the discharge burnup, are calculated. Results of the physical parameters all satisfy the relevant design requirements, which means the proposed scheme is safe and reliable and can provide support for the fuel management of HTR-PM in the future.
\end{abstract}

\section{Introduction}

Modular high-temperature gas-cooled reactor (HTGR) is a kind of safe and advanced nuclear energy system, which can efficiently provide electric power and high-temperature process-heat. On February 15, 2008, Chinese State Council approved the implementation plan of HTGR demonstration project. The goal is to build a $200 \mathrm{MW}_{\mathrm{e}}$ demonstration plant, named high-temperature gas-cooled reactor-pebblebed module (HTR-PM), as the first one meeting the safety standards of Generation-IV reactors in the world [1].

At present, the physical design of the equilibrium core of HTR-PM has been finished by Institute of Nuclear and New Energy Technology (INET) of Tsinghua University, which includes that the fuel spheres with $8.5 \%$ enrichment are adopted, pass through the core for 15 times, and reach the average discharge burnup of $90000 \mathrm{MWd} / \mathrm{tU}$. The emphasis in work is transferred to determine the scheme of the initial core and running-in phase.

From the initial core with fresh fuel, HTR-PM needs to take years of continuous refueling and burnup to reach the stable equilibrium core. Spending this time safely is the important research content of in-core fuel management and is an actual technical problem in pressing need of solution. There are many possible schemes for the initial core and running-in phase, and many factors need to be considered in the process of scheme evaluation and analysis. Before HTR-PM, some design schemes of pebble-bed reactors have been proposed, including THTR-300 [2] from Hochtemperatur-Kernkraftwerk GmbH in Germany, HTR10 [3] from Tsinghua University in China, HTR-Module [4] from Siemens AG in Germany, and PBMR [5] from PBMR (Pty) Ltd. in South Africa. Although the detailed design schemes of the initial core and running-in phase are different from each other, the common experience of these pebble-bed reactors shows that the fuel enrichment and the ratio of fuel spheres to graphite spheres are important.

In this paper, the physical model and simulation tool of HTR-PM are described in Section 2. The physical considerations of the initial core and running-in phase are, respectively, described in Sections 3 and 4. Then a typical scheme of the initial core and running-in phase is proposed and analyzed in Section 5. In the last section, a comprehensive comment is presented. 
TABLE 1: Main design parameters of HTR-PM.

\begin{tabular}{lcc}
\hline Parameters & Units & Values \\
\hline Reactor total thermal power & $\mathrm{MW}_{\text {th }}$ & $2 \times 250$ \\
Active core diameter & $\mathrm{m}$ & 3 \\
Equivalent active core height & $\mathrm{m}$ & 11 \\
Primary helium pressure & $\mathrm{MPa}$ & 7 \\
Helium temperature at reactor inlet/outlet & ${ }^{\circ} \mathrm{C}$ & $250 / 750$ \\
Uranium loading per fuel sphere & $\mathrm{g}$ & 7 \\
Enrichment of fresh fuel spheres & $\%$ & 8.5 \\
Diameter of fuel spheres & $\mathrm{cm}$ & 6 \\
Number of fuel spheres in one reactor core & & 420,000 \\
Average discharge burnup & $\mathrm{GWd} / \mathrm{tU}$ & 90 \\
\hline
\end{tabular}

\section{Physical Model and Simulation Tool}

2.1. Physical Model. The main technical parameters of HTRPM are presented in Table 1.

HTR-PM uses helium as coolant and graphite as moderator as well as structural material. Its spherical fuel elements contain thousands of very small "coated particles" which are embedded in the graphite matrix. The pebble-bed design allows fuel spheres to constantly pass the core by gravity from an up direction to a down direction, which ensures that the operation mode of continuous fuel loading and discharging is available without shutting down the reactor.

HTR-PM adopts the cylindrical single zone core. The ceramic structures surrounding the reactor core consist of the inner graphite reflector and outer carbon brick layers, as shown in Figure 1. The whole ceramic internals are installed inside a metallic core barrel, which itself is supported by reactor pressure vessel (RPV). The metallic core barrel and the RPV are protected against high temperature from the core by the cold helium borings of the side reflector, which act like a shielding temperature screen.

HTR-PM adopts multipass refueling strategy. The fuel spheres drop into the reactor core from the central fuel loading tube and are discharged through a fuel extraction pipe at the core bottom. Subsequently, the discharged fuel spheres pass the burnup measurement facility one by one. Depending on their burnup, either they will be discharged and transported into the spent fuel storage tank when having reached their designed burnup, or they will be reinserted into the reactor to pass the core once again.

2.2. Simulation Tool. VSOP [6] code is developed by the Institute for Safety Research and Reactor Technology, Juelich Research Center, Germany. The code has been widely used for the design of the high-temperature reactor (HTR) with spherical fuel elements, such as THTR-300 and HTR-Module in Germany, PBMR in South Africa, and HTR-10 in China. The application of the code includes the setup of the reactor and of the fuel element, processing of cross sections, neutron spectrum evaluation, neutron diffusion calculation in two or three dimensions, fuel depletion, fuel shuffling, reactor control, and thermal hydraulics of steady states and transients. The code can simulate the reactor operation from the initial core towards the equilibrium core.

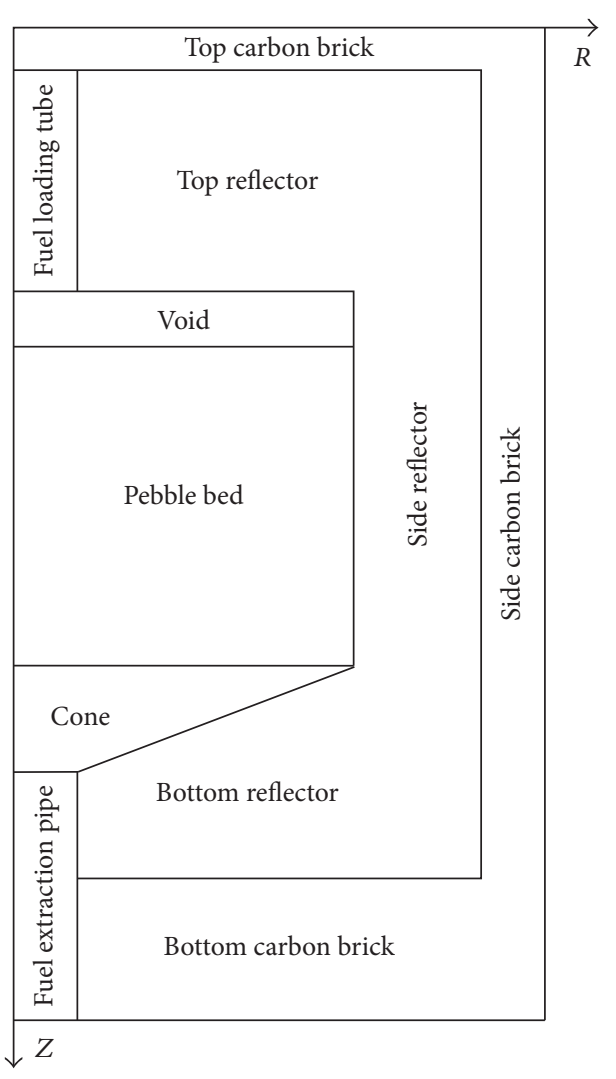

Figure 1: Geometric structure of HTR-PM core.

In VSOP code, to simulate the multipass refueling strategy of pebble-bed reactor, the reactor core is divided into different channels along the radial direction and different layers along the axial direction. Thus, lots of material regions are produced, which are the basic unit of neutron spectrum calculation. Then, each material region is divided into different fuel batches, which are the basic unit of fuel burnup calculation and fuel shuffling, and the number of fuel batches in material region is chosen as the designed times of fuel cycles. When the reactor is running, at the bottom of the core, the fuel batches with highest burnup will be discharged, and other fuel batches will be sent back to the top of the core, and meanwhile the bathes of fresh fuel will be loaded at the top of the core to keep the total quantity of fuel spheres stable. In the middle of the core, the fuel batches of each material region will be shuffled down to its neighbouring material region along the channel. Through the above method, the multipass refueling strategy of pebble-bed reactor can be well simulated. More detailed information can be seen in $[6,7]$.

Through the long-term validation and verification in the $10 \mathrm{MW}_{\mathrm{th}}$ high-temperature gas-cooled test reactor (HTR-10) as well as continuous updating by INET [8], VSOP code is thought to be capable of satisfying the requirements of HTRPM physical design.

\section{Physical Considerations of the Initial Core}

3.1. Loading Patterns of the Initial Core. The initial core is defined as the core that contains only the fresh fuel spheres, 
TABLE 2: The key physical parameters of typical volume fractions of fuel spheres in the initial core.

\begin{tabular}{lccc}
\hline $\begin{array}{l}\text { Volume fraction of fuel } \\
\text { spheres }\end{array}$ & $\begin{array}{c}\text { Fuel enrichment } \\
\text { needed }(\%)\end{array}$ & $\begin{array}{c}\text { Maximum power per } \\
\text { fuel sphere (kW/FS) }\end{array}$ & $\begin{array}{c}\text { Maximum fuel } \\
\text { temperature }\left({ }^{\circ} \mathrm{C}\right)\end{array}$ \\
\hline $1 / 15$ & 21.30 & 16.39 & 3401 \\
$5 / 15$ & 5.10 & 3.30 & 1093 \\
$10 / 15$ & 3.45 & 1.66 & 978 \\
$14 / 15$ & 3.10 & 1.18 & 951 \\
\hline
\end{tabular}

maybe with some graphite spheres, and that can reach the full power.

Due to the accumulation of fission products and the deep burnup of fuel spheres, in the equilibrium core, the average fuel enrichment over the core in HTR-PM is only $4.58 \%$, although the enrichment of fresh fuel spheres loaded in the core is $8.5 \%$. For the initial core almost without burnup, if only the fresh fuel spheres with $8.5 \%$ enrichment are loaded, the excess reactivity will be too big to control. To avoid this situation, some absorber balls containing boron need to be added in the core, or the quantity of fissile fuel in the core needs to be decreased, which can be achieved through adding some graphite spheres or decreasing the enrichment of the fuel spheres. As a result, the possible loading patterns of the initial core are formed as follows. (1) Fuel spheres with $8.5 \%$ enrichment or lower enrichment and graphite spheres are adopted; (2) fuel spheres with $8.5 \%$ enrichment or lower enrichment and absorber balls are adopted; (3) fuel spheres with $8.5 \%$ enrichment or lower enrichment and graphite spheres as well as absorber balls are adopted; (4) only fuel spheres with very low enrichment are adopted.

To determine the loading pattern of the initial core of HTR-PM, some important factors need to be considered, which are as follows.

(1) Enrichment of fuel spheres: if the initial core and equilibrium core both load the fuel spheres with $8.5 \%$ enrichment, the processes of fuel purchase, manufacture, and management can be simplified, according to the THTR-300 and HTR-10 experiences. Considering the requirement of excess reactivity control, not many fuel spheres will be loaded, which increases the power per fuel sphere and is disadvantageous for safety. HTR-Module and PBMR recommend adopting lowenrichment fuel spheres in the initial core, which can increase the quantity of fuel spheres in the core and decrease the power per fuel sphere. So, in HTR-PM, the low-enrichment fuel spheres are adopted.

(2) Absorber balls: another way to increase the quantity of fuel spheres loaded in the core and to decrease the power per fuel sphere is loading some absorber balls. In THTR-300 and HTR-Module, a small quantity of absorber balls is adopted. Unfortunately, experience of manufacture and application of absorber balls is lacking in China. Moreover, considering the big movement randomness of the small quantity of absorber balls in the core will increase the difficulty of fuel management; the absorber balls are not expected in the initial core of HTR-PM.
(3) Graphite spheres: the enrichment of fuel spheres loaded in the initial core can be increased if some graphite spheres are added, which can decrease the difference of enrichment between the initial core and equilibrium core to make the running-in phase more smooth and can avoid discharging fuel spheres at the beginning of running-in phase to improve the fuel utilization. In THTR-300 and HTR-10, significant amount of graphite spheres is loaded in the initial core, and this is also recommended by HTR-Module and PBMR. So, in HTR-PM, the graphite spheres are planned to be used.

3.2. Physical Characteristics of the Initial Core. HTR-PM adopts the scheme of mixing low-enrichment fuel spheres with graphite spheres as its loading pattern for the initial core, in which the ratio of fuel spheres to graphite spheres needs to be determined. In Table 2, the key physical parameters of several typical volume fractions of fuel spheres in the initial core are given.

From Table 2, the following physical characteristics can be seen. (1) If the volume fraction of fuel spheres in the initial core is very low, the enrichment of fuel spheres needs to be very high to keep the reactor critical, even higher than $8.5 \%$ adopted in the equilibrium core, which is unpractical. Besides this, the small value of volume fraction of fuel spheres in the initial core means that there are few fuel spheres to share the operation power, causing big values of power per fuel sphere and fuel temperature, which is not safe. Considering these problems, the volume fraction of fuel spheres in the initial core should be as high as possible. (2) When the volume fraction of fuel spheres in the initial core is very high, the enrichment of fuel spheres needs be very low. In the subsequent running-in phase, if the fuel spheres with $8.5 \%$ enrichment adopted in the equilibrium core are directly added to replace the discharged fuel spheres with low enrichment, the difference of enrichment will cause big value of power per fuel sphere in the running-in phase, considering the fact that the fuel spheres with high enrichment will produce more power. To avoid this situation, one or more kinds of fuel spheres with medium enrichment are needed, but this could bring additional problems for fuel purchase, manufacture, and management. From these respects, the volume fraction of fuel spheres in the initial core should be as low as possible. (3) In conclusion, if the volume fraction of fuel spheres in the initial core is medium, the above advantages and disadvantages can be well balanced, and good physical characteristics can be expected. 


\section{Physical Considerations of the Running-In Phase}

There are some basic considerations for the refueling patterns of the running-in phase of HTR-PM.

(1) The process of replacing the graphite spheres and the process of replacing the low-enrichment fuel spheres loaded in the initial core should be separated, which is beneficial for stabilizing the running-in phase and simplifying the operation of the fuel handling and storage system (FHSS). This is recommended by HTR-Module and PBMR.

(2) The process of replacing the graphite spheres with fuel spheres should be performed at the beginning of running-in phase, which can quickly increase the quantity of fuel spheres in the core and thus decrease the power per fuel sphere and can avoid discharging fuel spheres at the beginning of running-in phase to improve fuel utilization. This is performed in THTR300 and HTR-10 and is recommended by HTRModule and PBMR.

(3) For simplifying the processes of fuel purchase, manufacture, and management, it is better to use as few kinds of fuel spheres in enrichment as possible in the running-in phase. In HTR-Module, three kinds of fuel spheres are recommended, including the low-enrichment fuel spheres loaded in the initial core, the medium-enrichment fuel spheres used in the running-in phase, and the high-enrichment fuel spheres adopted in the equilibrium core.

(4) In the running-in phase, the times of fuel cycles should be as many as possible, which can smooth the power distribution and burnup distribution along the axial direction of the core [9], but the refueling rate should be controlled in the capability of FHSS.

\section{Physical Analysis of a Typical Scheme of the Initial Core and Running-In Phase}

5.1. Description of the Scheme. Based on the above physical considerations of the initial core and running-in phase, a typical scheme for HTR-PM is proposed as follows.

(1) The initial core is made up of the fuel spheres with $4.1 \%$ enrichment and the graphite spheres. The volume fraction of fuel spheres in the core is $7 / 15$.

(2) The running-in phase is divided into three continuous processes. In the first process, $3 / 8$ of the graphite spheres are replaced by the fuel spheres with $4.1 \%$ enrichment. When the first process is ended, the volume fraction of fuel spheres in the core is improved to $10 / 15$. In the second process, the remaining graphite spheres are replaced by the fuel spheres with $4.1 \%$ enrichment. When the second process is ended, they are full of fuel spheres with $4.1 \%$ enrichment in the core. The last process includes 15 fuel cycles. In each cycle, $1 / 15$ of the fuel spheres with $4.1 \%$ enrichment are

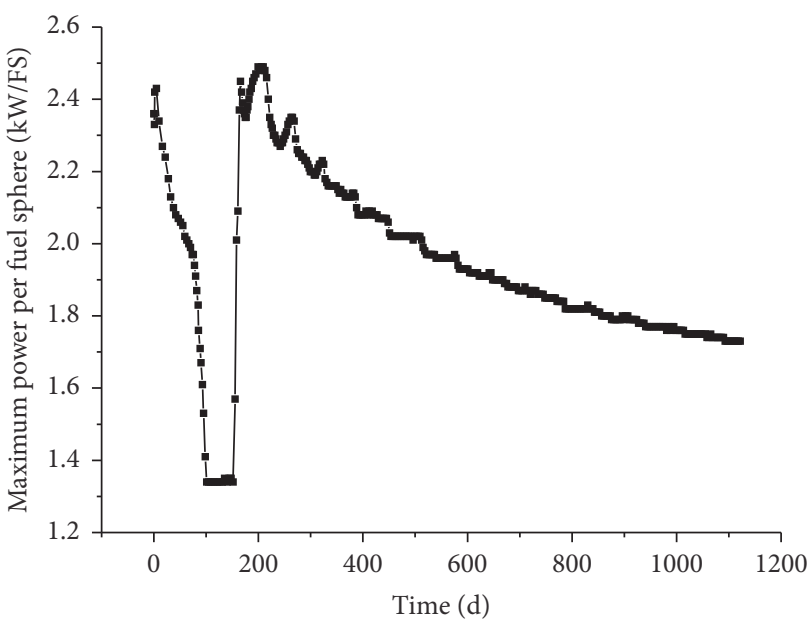

FIgURE 2: Maximum power per fuel sphere changing with time.

replaced by the fuel spheres with $8.5 \%$ enrichment. When this process is ended, they are full of fuel spheres with $8.5 \%$ enrichment in the core, and the status is close to the equilibrium core.

5.2. Physical Characteristics of the Scheme. The above scheme is simulated with VSOP code, and the key physical parameters, such as the maximum power per fuel sphere, the maximum fuel temperature, the refueling rate, and the discharge burnup, are calculated.

Results of the maximum power per fuel sphere in the running-in phase are shown in Figure 2.

In the running-in phase, the maximum power per fuel sphere reaches $2.49 \mathrm{~kW} / \mathrm{FS}$, which is higher than that in the equilibrium core $(1.81 \mathrm{~kW} / \mathrm{FS})$, but there is still some margin compared with the safety limit value $(3.5 \mathrm{~kW} / \mathrm{FS})$.

There are two obvious peak values of the maximum power per fuel sphere in the running-in phase. The first one appears at the beginning of running-in phase. That is mainly because the fuel spheres loaded in the core are very few at that time, and the peak value becomes smaller along with adding fuel spheres. The second peak value appears at the time of adding the fuel spheres with $8.5 \%$ enrichment. That is mainly because the enrichment of the new added fuel spheres is much higher than the depleted fuel spheres, which means the new added fuel spheres share a great part of operation power.

Results of the maximum fuel temperature in the runningin phase are shown in Figure 3.

In the running-in phase, the maximum fuel temperature reaches $1022^{\circ} \mathrm{C}$, which is higher than that in the equilibrium core $\left(932^{\circ} \mathrm{C}\right)$, but there is still some margin compared with the safety limit value $\left(1200^{\circ} \mathrm{C}\right)$.

Results of the refueling rate in the running-in phase are shown in Figure 4.

In the running-in phase, the refueling rate is controlled in the capability of FHSS, that is, lower than $12000 \mathrm{FS} / \mathrm{d}$. It can be seen that, at the beginning of running-in phase, the refueling rate is very high, which is because the fissile fuel loaded in the core is much less than that in the equilibrium 


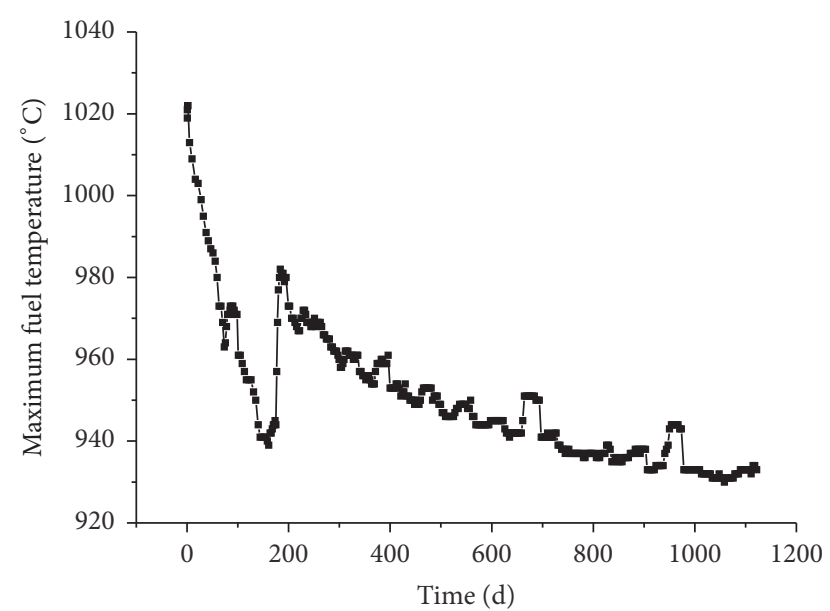

Figure 3: Maximum fuel temperature changing with time.

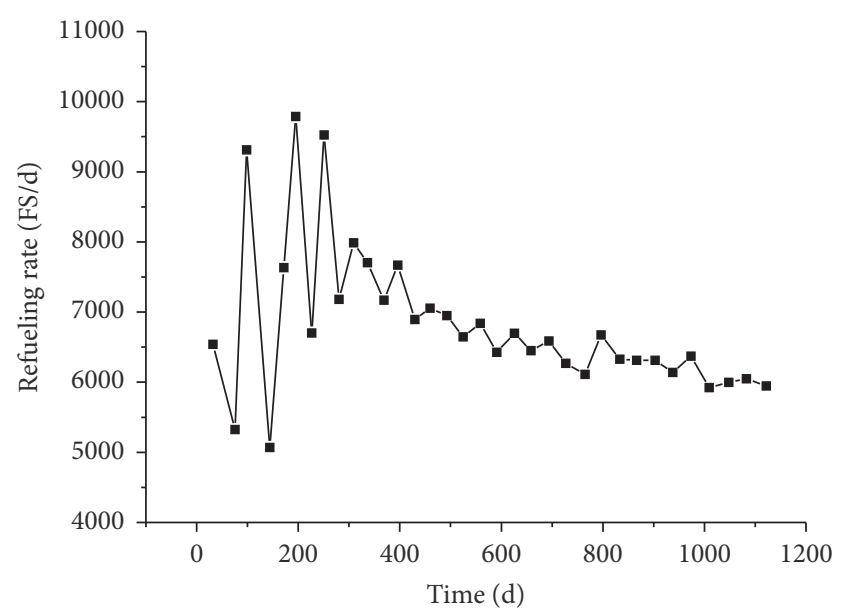

FIGURE 4: Refueling rate changing with time.

core. It makes the reactivity decrease with burnup faster than that in the equilibrium core, and thus higher refueling rate is needed to maintain normal operation of the reactor. And at the beginning of running-in phase, the refueling rate fluctuates widely, which is because the power distribution and burnup distribution along the axial direction of the core have not been flattened well during this period. But, with the development of the running-in phase, the composition and burnup in the core are getting more and more welldistributed, resulting in smaller fluctuation of the refueling rate.

Results of the discharge burnup in the running-in phase are shown in Figure 5.

In the running-in phase, the discharge burnup is lower than the safety limit value $(100000 \mathrm{MWd} / \mathrm{tU})$. At 143.9 days, all the graphite spheres are discharged, and then the depleted low-enrichment fuel spheres start to be discharged and the initial discharge burnup is $16281 \mathrm{MWd} / \mathrm{tU}$. The discharge burnup climbs to $57342 \mathrm{MWd} / \mathrm{tU}$ with operation of the reactor. At 1118.2 days, all of the depleted low-enrichment fuel spheres are discharged, and then the depleted high-enrichment fuel

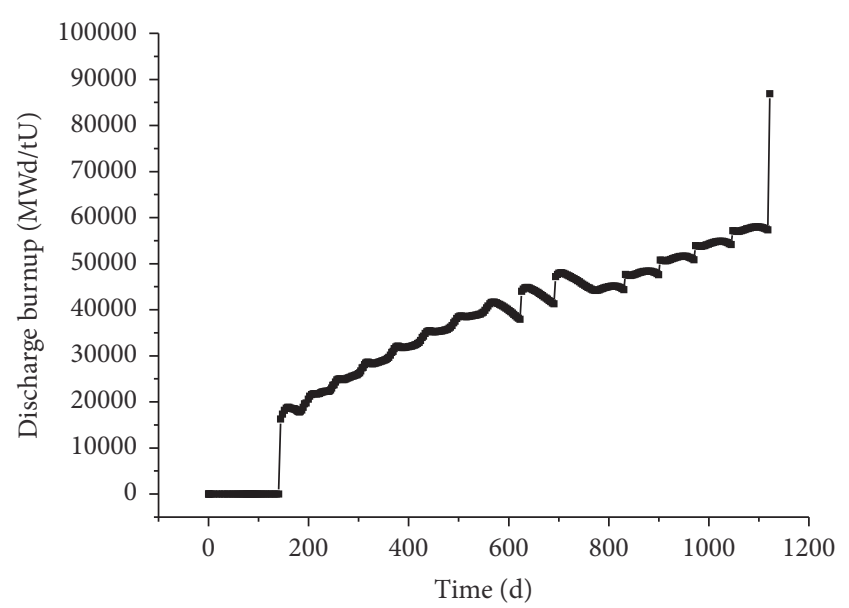

FIGURE 5: Discharge burnup changing with time.

spheres start to be discharged and the discharge burnup is close to that in the equilibrium core $(90000 \mathrm{MWd} / \mathrm{tU})$.

The uncertainty quantification of the calculation results of VSOP code for HTR-PM design has been performed by INET, involving the uncertainties from nuclear data, pebble flow [10], filling fraction of pebble bed [11], uranium loading per fuel sphere, and some thermal-hydraulic parameters. The corresponding influence on the key physical parameters, such as $k_{\text {eff }}$, power density, power peak, axial offset of power (AO), fuel temperature, and fuel burnup, is detailedly evaluated. The results show that the maximum uncertainty for the situation of normal operation is less than $1.0 \%$ [12].

As to this paper, the calculation results of VSOP code for the proposed scheme of the initial core and running-in phase are $10 \% \sim 20 \%$ lower than the safety limit values. So, after taking the corresponding uncertainties into account, the safety margins are still enough.

\section{Conclusions}

In this paper, some physical considerations for the fuel management of the initial core and running-in phase of HTR$\mathrm{PM}$ are given, including the advantages and disadvantages of different loading patterns of the initial core and different refueling patterns of the running-in phase. Then a typical scheme of the initial core and running-in phase of HTR-PM is proposed, in which one kind of graphite sphere and two kinds of fuel spheres in enrichment are used. Firstly, the graphite spheres are replaced by low-enrichment fuel spheres through two fuel cycles. When all the graphite spheres are discharged, the low-enrichment fuel spheres start to be replaced by high-enrichment fuel spheres through fifteen fuel cycles. When all the low-enrichment fuel spheres are discharged, the running-in phase is ended and the equilibrium core is established. The above scheme of the initial core and runningin phase is simulated with VSOP code, and some key physical parameters, such as the maximum power per fuel sphere, the maximum fuel temperature, the refueling rate, and the discharge burnup, are calculated. Results of the physical parameters all satisfy the relevant design requirements, which 
means the proposed scheme is safe and reliable. In the future, more detailed physical analysis of the scheme will be performed, especially for the accident conditions.

\section{Conflicts of Interest}

The authors declare that there are no conflicts of interest regarding the publication of this paper.

\section{Acknowledgments}

The authors would like to express their gratitude for the support by National Natural Science Foundation of China (Project 11605058 and Project 11375099) and Chinese National S\&T Major Project ZX06901.

\section{References}

[1] Z. Zhang, Z. Wu, D. Wang et al., "Current status and technical description of Chinese $2 \times 250$ MWth HTR-PM demonstration plant," Nuclear Engineering and Design, vol. 239, no. 7, pp. 12121219, 2009.

[2] R. Bäumer, I. Kalinowski, E. Röhler, J. Schöning, and W. Wachholz, "Construction and operating experience with the 300-MW THTR nuclear power plant," Nuclear Engineering and Design, vol. 121, no. 2, pp. 155-166, 1990.

[3] Z. Wu, D. Lin, and D. Zhong, "The design features of the HTR10," Nuclear Engineering and Design, vol. 218, no. 1-3, pp. 25-32, 2002.

[4] G. H. Lohnert, "Technical design features and essential safetyrelated properties of the HTR-module," Nuclear Engineering and Design, vol. 121, no. 2, pp. 259-275, 1990.

[5] J. F. M. Slabber and E. J. Mulder, "Loading of the PBMR initial core and the transition into the equilibrium core," in Proceedings of the International Congress on Advances in Nuclear Power Plants (ICAPP '05), Seoul, Republic of Korea, May 2005.

[6] H. J. Rütten, K. A. Haas, H. Brockmann, and W. Scherer, V.S.O.P. (99/05) Computer Code System for Reactor Physics and Fuel Cycle Simulation, Forschungszentrum Jülich GmbH, 2005.

[7] Z. Wu and X. Jing, "Fuel management of HTR-10," Journal of Tsinghua University (Science and Technology), vol. 41, no. 4-5, pp. 120-123, 2001 (Chinese).

[8] International Atomic Energy Agency, "Evaluation of high temperature gas cooled reactor performance: benchmark analysis related to the PBMR-400, PBMM, GT-MHR, HTR-10 and the ASTRA critical facility," Tech. Rep. IAEA-TECDOC-1694, 2013.

[9] B. Ling and Y. Yang, Theory of Nuclear Reactor Engineering, Atomic Press, Beijing, China, 1982 (Chinese).

[10] H. Chen, L. Fu, G. Jiong, S. Ximing, and W. Lidong, "Quantitative analysis of uncertainty from pebble flow in HTR," Nuclear Engineering and Design, vol. 295, pp. 338-345, 2015.

[11] H. Chen, L. Fu, G. Jiong, and W. Lidong, "Uncertainty and sensitivity analysis of filling fraction of pebble bed in pebble bed HTR," Nuclear Engineering and Design, vol. 292, pp. 123132, 2015.

[12] C. Hao, Uncertainty analysis in modeling of pebble bed HTR [ph.D. dissertation], Tsinghua University, Beijing, China, 2014 (Chinese). 


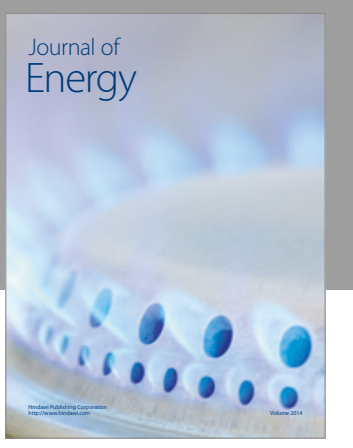

Journal of

Industrial Engineering
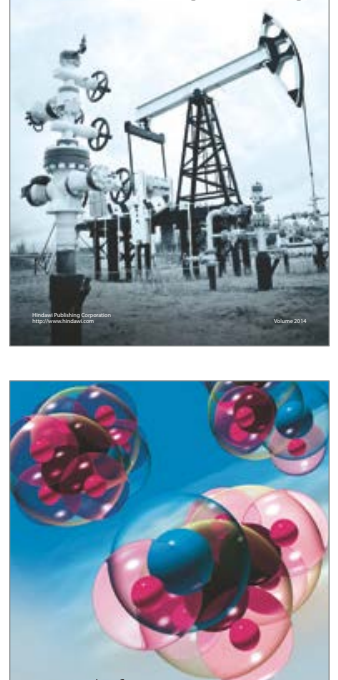

Fuels
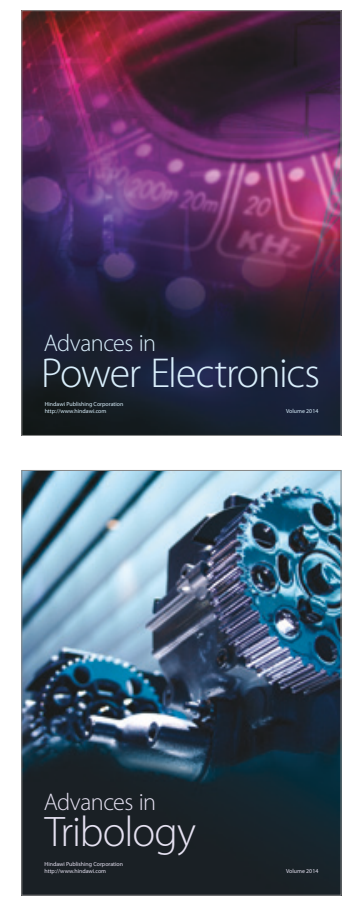
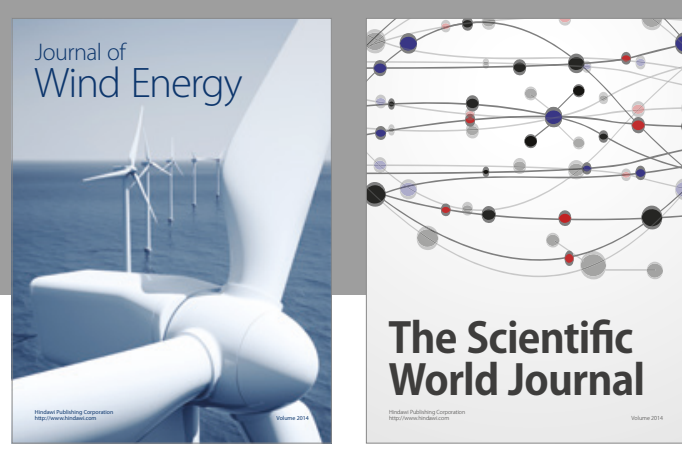

The Scientific World Journal
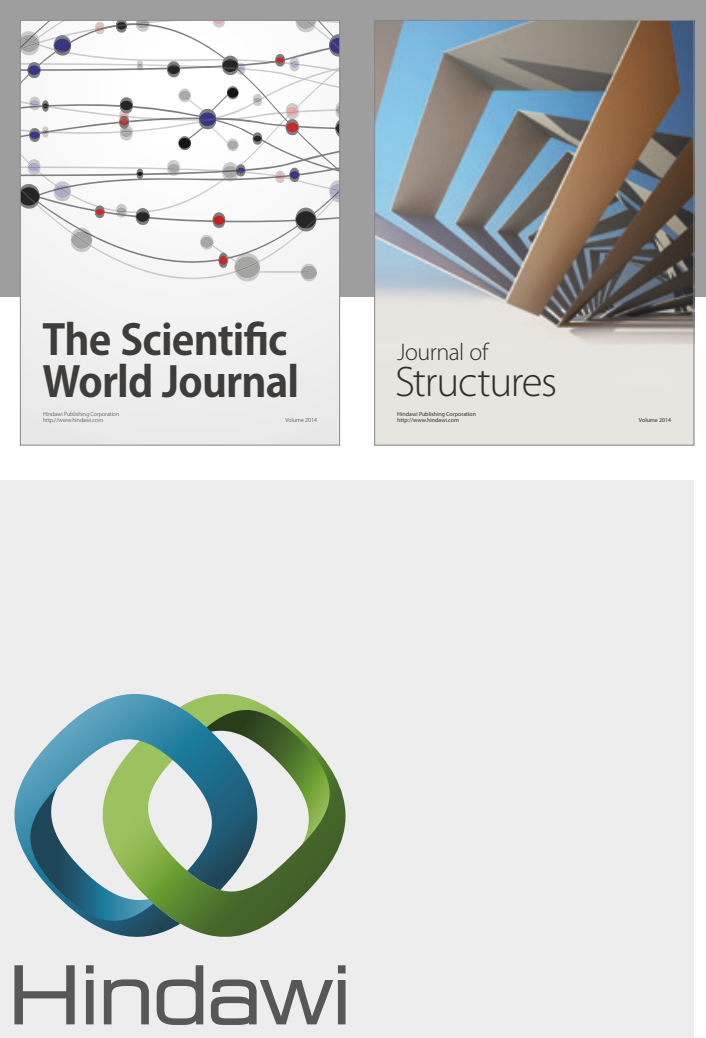

Submit your manuscripts at

https://www.hindawi.com
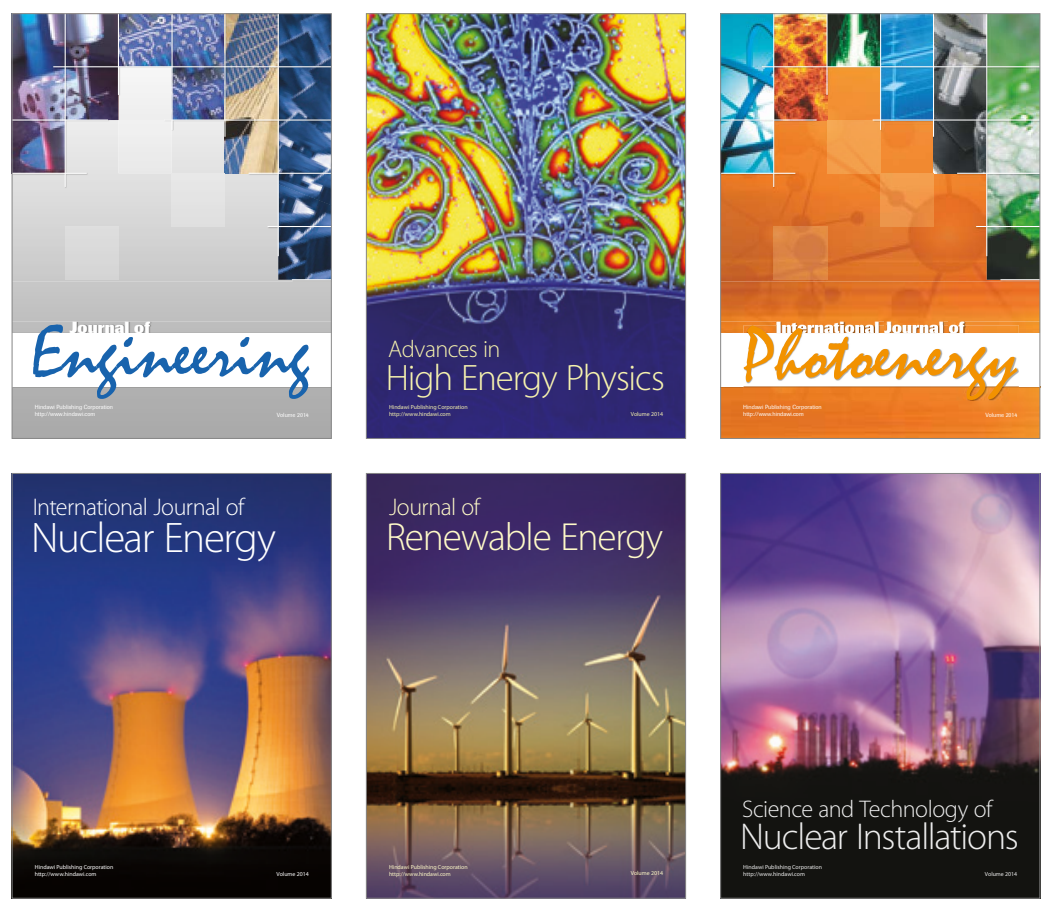

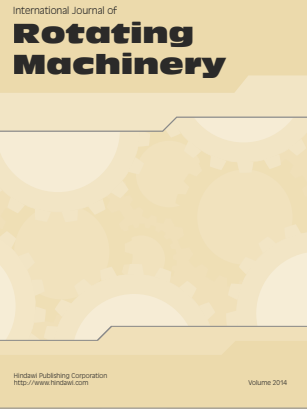

Journal of

Petroleum Engineering

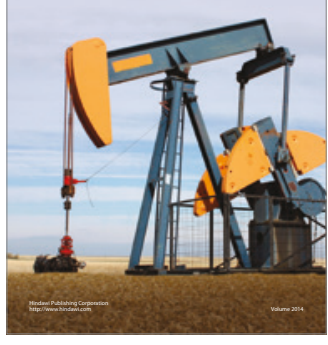

Journal of
Solar Energy
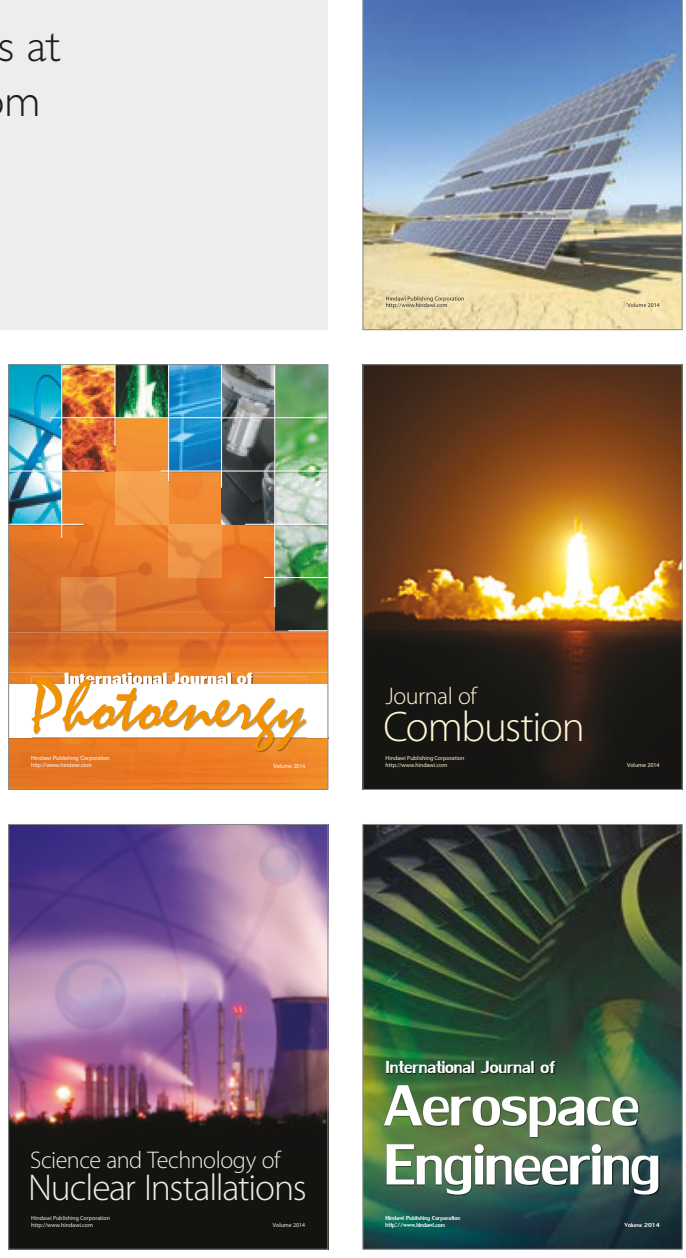